\title{
Methodology for the Selection and Sizing of an Isolated MicroGrid Based on Economic Criteria
}

\author{
G. Catuogno, Senior Member, IEEE, L. Torres, L. Proietti, and G. Garcia, Senior Member, IEEE.
}

\begin{abstract}
The present work proposes a methodology for the selection and sizing of rural microgrids in small communities improving the traditional solution of the generator group with the incorporation of renewable energies. The methodology includes four stages, the data collection, the definition of the system, the optimization and the selection of the best option. The HOMER Pro software is used to perform economic optimization. Finally, the rural school of Santa Bárbara in the Puertas del Sol area in the department of San Martín of the Province of San Luis, Argentina, is presented as a case study.
\end{abstract}

Index Terms-Design methodology, Economic criteria, Isolated microgrid, Solar Energy, Wind Energy.

\section{INTRODUCCIÓN}

$\mathrm{L}^{\wedge}$ A electrificación en zonas remotas o rurales se ha realizado tradicionalmente mediante dos alternativas, la extensión de la red eléctrica, o la incorporación de grupos electrógenos de mediana o pequeña potencia. Con el precio del petróleo alcanzando sus niveles más altos y los altos costos de expansión de la línea de transmisión, combinado con la necesidad de reducir las emisiones de dióxido de carbono, las energías renovables se han convertido en una alternativa importante como proveedor de energía en los sistemas rurales $[1,2]$. Si bien el costo inicial de la energía de fuentes convencionales es menor que a partir de fuentes de energía renovables, la combinación de suministro de energía renovable y grupo electrógeno puede reducir el costo de la energía [3].

Sin embargo, la correcta estimación del tipo de sistema de energía renovable debe hacerse bajo técnicas de optimización. Es por lo anterior que el diseño óptimo de sistemas híbridos de energía renovable es un tema candente y hay una gran cantidad de literatura dedicada a este tema. El problema de diseño mencionado a formularse está relacionado con la determinación de la configuración óptima del sistema de potencia y la ubicación óptima, el tipo y el tamaño de las unidades de generación instaladas, de modo que el sistema cumpla con los requisitos de carga a un costo mínimo [4].

Numerosos trabajos de optimización para el dimensionamiento de sistemas híbridos han sido reportadas en la literatura tales como algoritmo genético (AG) [5], [6],

This work was supported in part by the National Council of Scientific and Technical Research (CONICET) in Argentina.

G. Catuogno are L. Torres are with the National University of San Luis, Argentina (grcatu@ieee.org, torresluisraul.ing@gmail.com).

L. Proietti in co-founder of NGO 500RPM, Buenos Aires, Argentina, (luciana@500rpm.org).

G. Garcia is with the National University of Rio Cuarto, Rio Cuarto, Argentina (ggarciasapino@gmail.com). inteligencia artificial (IA), control predictivo basado en modelo (MPC) [7] y algoritmos de enjambre de partículas evolutivas [8]. Además, se han desarrollado y utilizado ampliamente diversas herramientas comerciales de dimensionamiento que serán utilizadas en este trabajo [9].

Para planificar la microrred en áreas rurales considerando fuentes de energía renovables, se requiere la definición de varios factores, tales como, las mejores fuentes de energía renovable para ser utilizadas, el número y la capacidad de estas fuentes de generación, el consumo eléctrico, el costo total del sistema, la cantidad de las emisiones que se pueden eliminar, la distancia desde el punto de conexión de la red más cercana, el exceso de energía, la carga no satisfecha, los precios del combustible, las diferentes cargas y los sistemas conectados a la red. Además, en muchos países, los gobiernos alientan firmemente a los planificadores de las microrredes a que estén motivados para invertir en el sector de las energías renovables.

Con base en lo anterior, los programas de simulación son las herramientas más comunes para evaluar el rendimiento de los sistemas híbridos de energía renovable. Actualmente, hay muchos programas de software disponibles que se pueden descargar de los sitios web de varios laboratorios de investigación y universidades. Al utilizar los programas de simulación mencionados, se puede encontrar la configuración óptima al comparar el rendimiento y el costo de producción de energía de las diferentes configuraciones del sistema. En [10] se realiza una comparación detallada sobre herramientas de software disponibles comercialmente para la evaluación del rendimiento de los sistemas híbridos de energía renovable.

Uno de los programas de dimensionamiento más difundidos para sistemas híbridos es el HOMER Pro desarrollado por National Renewable Energy Laboratory (NREL), Estados Unidos [11]. Este software incluye varios modelos de componentes de energía, como generadores fotovoltaicos (PV), turbinas eólicas, centrales hidroeléctricas, baterías, grupo electrógeno y otros generadores a combustible, unidades de electrólisis y pilas de combustible, y evalúa opciones adecuadas considerando el costo y la disponibilidad de recursos energéticos. La conexión a la red también se considera en el procedimiento de diseño HOMER Pro.

El software requiere información inicial que incluye recursos de energía, restricciones económicas y técnicas, requisitos de almacenamiento de energía y estrategias de control del sistema. También, se requieren entradas como tipo de componente, costos iniciales (CI), costo de reemplazo (CR), costos de operación y mantenimiento (CO\&M), eficiencia, vida operativa, etc [12].

Este trabajo propone una metodología para la selección y dimensionamiento de una microrred aislada, basada en un 
análisis económico, resultando en un sistema sustentable que optimiza la relación costo/operación a largo plazo.

El trabajo está organizado de la siguiente manera, la Sección 2 presenta la metodología de diseño, la Sección 3 discute brevemente el caso de estudio bajo consideración y los datos de entrada del sistema. La Sección 4 expone los resultados obtenidos mediante la metodología, y finalmente la Sección 5 ofrece las conclusiones del trabajo realizado.

\section{METODOLOGÍA DE DISEÑO}

La metodología propuesta se basa en cuatro etapas bien definidas que se presentan en la Fig. 1 y se describen en las siguientes subsecciones $[13,14]$.

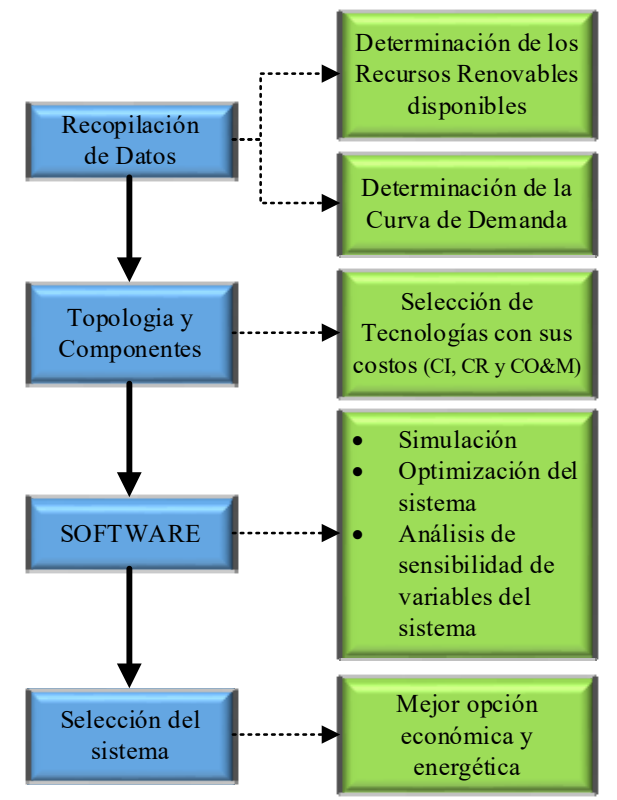

Fig. 1. Diagrama de la metodología de diseño.

\section{A. Recopilación de Datos}

En esta etapa se recopilan todos los datos relevantes del lugar. Se estudian las fuentes de energías ya instaladas, la disponibilidad de recursos renovables, la cotización de la extensión de la red eléctrica y se determina las características de consumo eléctrico de los usuarios del lugar.

La disponibilidad de los recursos renovables se determina por un pronóstico a largo plazo de la variable en interés (velocidad del viento, irradiancia, etc.). Esta información puede ser obtenida directamente de sitios meteorológicos (nacional, internacional, etc.) o por predicciones (usando regresión lineal, Deep Learning, etc.) basados en datos históricos de la variable en el lugar considerado $[15,16]$.

La curva de demanda se obtiene mediante la información de algún sistema de registro de potencia eléctrica local (medidores de electricidad) o por el cálculo de consumo eléctrico en base a la potencia y el número de las cargas, en función del tiempo de consumo de los usuarios (mediciones de campo, encuestas de consumo, etc.).

Los datos recopilados se evalúan según,

- Disponibilidad de recursos renovables: valores de irradiancia y velocidad del viento mensual, potencial hídrico, producción de biomasa, etc.
- Disponibilidad de recursos no renovables: grupo electrógeno, red.

- Las condiciones de carga de los usuarios: número de usuarios, distancia entre casas, consumos típicos, consumo reprimido, etc.

- Las condiciones económicas: posibilidades de financiamiento, índice de necesidades básicas insatisfechas y condiciones de acceso.

\section{B. Selección de Topología y Componentes}

La selección de componentes se basa en los recursos renovables disponibles obtenidos de la recopilación de datos. Se deben identificar los costos iniciales, de reemplazo y los costos de operación y mantenimiento, como también la eficiencia y vida operativa del componente.

Debe tenerse en cuenta que la complejidad de la topología y las características de los componentes de la microrred, definirán el nivel técnico para su operación y mantenimiento básico, y por tanto, el tipo de capacitación que se deberá impartir a los usuarios para un funcionamiento seguro.

\section{Simulación Computacional (optimización)}

En esta subsección se realiza la simulación computacional que se encarga de realizar la optimización en función de criterios económicos. Se propuso el software HOMER Pro por ser unos de los más difundidos en el diseño de microrredes aisladas.

HOMER Pro realiza tres tareas principales: simulación, optimización y análisis de sensibilidad. En el proceso de simulación, HOMER Pro modela el rendimiento de una configuración particular del sistema de micro potencia cada hora del año para determinar su factibilidad técnica y el costo del ciclo de vida. En el proceso de optimización, HOMER Pro simula muchas configuraciones diferentes del sistema en busca de la que satisfaga las restricciones técnicas al menor costo del ciclo de vida [17]. En el proceso de análisis de sensibilidad, HOMER Pro realiza múltiples optimizaciones bajo un rango de supuestos de entrada para medir los efectos de la incertidumbre o los cambios en las entradas del modelo. La optimización determina el valor óptimo de las variables sobre las que el diseñador del sistema tiene control, como la combinación de componentes que conforman el sistema y el tamaño o la cantidad de cada uno. Finalmente, el análisis de sensibilidad ayuda a evaluar los efectos de la incertidumbre o los cambios en las variables sobre las cuales el diseñador no tiene control, como la velocidad promedio del viento o el precio futuro del combustible.

Los dos principales indicadores económicos considerados en el presente análisis son el costo neto actual total $\left(\mathrm{C}_{\mathrm{NPC}}\right)$ y el costo nivelado de la energía (COE). El $C_{N P C}$ es más confiable en comparación con $\mathrm{COE}$ como parámetro económico, ya que el valor de $\mathrm{COE}$ es arbitrario en cierta medida, mientras que $\mathrm{C}_{\mathrm{NPC}}$ se deriva de un concepto matemático. Las desventajas del software son los altos tiempos de cálculo relativos, y las limitaciones en la optimización ya que el único objetivo a minimizar es el costo actual neto $\left(\mathrm{C}_{\mathrm{NPC}}\right)$ a diferencia de otras técnicas multicriterio [18].

Para su cálculo en necesario determinar el costo capital anualizado de cada componente que puede determinarse de la siguiente manera, 


$$
\mathrm{C}_{\mathrm{acap}}=\mathrm{C}_{\mathrm{cap}} \cdot \mathrm{CRF} \text {, }
$$

donde $\mathrm{C}_{\text {cap }}$ es el costo del capital inicial de un componente al inicio del proyecto y el CRF es el factor de recuperación de capital, dado por la siguiente ecuación,

$$
\mathrm{CRF}=\frac{\mathrm{i}(1+\mathrm{i})^{\mathrm{N}}}{(1+\mathrm{i})^{\mathrm{N}}-1}
$$

donde $\mathrm{N}$ e i son la vida útil del sistema y la tasa de interés real anual. La tasa de descuento real o también llamada tasa de interés real se calcula mediante la ecuación de Fisher de la siguiente manera $[19,20]$,

$$
i=\frac{i^{\prime}-\mathrm{f}}{1+\mathrm{f}},
$$

donde $i$ 'es la tasa de interés nominal (TIN) que a diferencia de la tasa de interés real, no tiene en cuenta la inflación $\mathrm{f}$ esperada para el periodo del proyecto $(\$)$.

A partir de estos cálculos, es posible obtener el costo neto actual, con el objetivo de encontrar el menor valor entre todas las combinaciones posibles que se dan en el espacio de búsqueda $\left(\min \left(C_{N P C}\right)\right.$.

El costo neto actual total de cada configuración se puede calcular de la siguiente manera,

$$
\mathrm{C}_{\mathrm{NPC}}=\frac{\mathrm{TAC}}{\mathrm{CRF}}
$$

donde TAC es el costo total anualizado (suma de todos los costos e ingresos anualizados de cada componente del sistema) que es,

$$
\mathrm{TAC}=-\left[-\mathrm{C}_{\mathrm{acap}}{ }^{-} \sum_{\mathrm{j}=1}^{\mathrm{n}} \mathrm{C}_{\mathrm{O} \& \mathrm{M}, \mathrm{j}^{-}} \mathrm{C}_{\mathrm{f}^{-}} \sum_{\mathrm{j}=1}^{\mathrm{n}} \mathrm{C}_{\text {Reemp }, \mathrm{j}}+\sum_{\mathrm{j}=1}^{\mathrm{n}} \mathrm{C}_{\text {Recup }, \mathrm{j}}\right] \text {, (5) }
$$

donde $n$ es el número de todos los componentes del sistema, $\mathrm{C}_{\mathrm{O \& M}, \mathrm{j}}$ es el costo anual de operación y mantenimiento para el componente $\mathrm{j}$ del sistema, $\mathrm{C}_{\mathrm{f}}$ es el costo total anual de combustible que es obtenido del producto de la cantidad de litros de combustible utilizados y el costo anualizado de combustible, $\mathrm{C}_{\mathrm{Reemp}, \mathrm{j}}$ es el costo de reemplazo anualizado para el componente $\mathrm{j}$ del sistema $\mathrm{y} \mathrm{C}_{\text {Recup }}$ es el costo de recuperación, que representa el valor restante de un componente del sistema de energía al final de la vida útil del proyecto, que sufre una depreciación lineal, lo que significa que es directamente proporcional a la vida restante. Además, se basa en el costo de reposición más que en el costo de capital inicial se puede expresar de la siguiente manera [20],

$$
\mathrm{C}_{\text {Recup }}=\mathrm{C}_{\text {Reemp }} \frac{\mathrm{R}_{\mathrm{R}}}{\mathrm{R}_{\mathrm{C}}} \text {, }
$$

donde $C_{\text {Reemp }}$ representa el costo de reemplazo $(\$), R_{C}$ la vida útil del componente en años y $R_{R}$ la vida restante del componente al final de la vida del proyecto que viene dada por,

$$
R_{R}=R_{C}-\left(R_{P r}-R_{R p}\right)
$$

donde $R_{P r}$ es el tiempo de vida del proyecto (en años) y $R_{R p}$ es la duración del costo de reemplazo definida como,

$$
\mathrm{R}_{\mathrm{Rp}}=\mathrm{R}_{\mathrm{C}} \cdot \mathrm{Z}\left(\frac{\mathrm{R}_{\mathrm{Pr}}}{\mathrm{R}_{\mathrm{C}}}\right)
$$

donde $\mathrm{Z}$ significa que se toma el numero entero del cociente. Nótese que en (5) no se consideraron los costos e ingresos por la compra y venta de potencia de la red eléctrica ya que en este manuscrito solo se consideran microrredes aisladas.

Finalmente, el costo nivelado de la energía (COE) se define como el costo promedio por $\mathrm{kWh}$ de energía eléctrica útil producida por el sistema. El COE representa un costo constante por unidad de generación, que se calcula para comparar el costo de diferentes fuentes de generación y tecnologías. El COE se calcula dividiendo el costo anualizado de producir electricidad por la carga eléctrica total servida. La ecuación para el COE es la siguiente,

$$
\mathrm{COE}=\frac{\mathrm{TAC}}{\mathrm{E}_{\text {Generada }}},
$$

donde $\mathrm{E}_{\mathrm{Generada}}$ es la sumatoria de toda la energía generada o producida en el año por cada fuente de generación.

Durante la simulación, el software se encarga de realizar todas las combinaciones posibles de los sistemas de generación definidos $\mathrm{y}$ en los espacios de estado determinados, el resultado es una lista de todas las posibles combinaciones ordenadas de menor a mayor en función del valor del $\mathrm{C}_{\mathrm{NPC}}$, determinando que la de menor costo actual neto es la óptima.

\section{Selección del Sistema}

Finalmente, a partir de la optimización realizada por el software, se obtendrá la mejor solución basada en criterios económicos a lo largo de los años que dure el proyecto.

En esta instancia es muy importante el criterio de los diseñadores basados en experiencias previas, ya que posiblemente la mejor solución contemple varios sistemas renovables, lo que dificultaría la operación y mantenimiento para los pobladores, $u$ otros motivos que tengan en cuenta aspectos sociales.

\section{CASO DE ESTUDIO}

Se propone analizar como caso de estudio la escuela rural Santa Bárbara en el departamento General San Martin en la Provincia de San Luis. El camino a la escuela es por la ruta provincial RP-2 donde los últimos 20 kilómetros son de un camino de ripio de difícil acceso no permitiendo que puedan circular vehículos urbanos.

En un radio de $10 \mathrm{~km}$ se encuentran las localidades más cercanas que son Libertador de San Martin y la localidad de Quines perteneciente al Departamento Ayacucho. Las 
principales características de la escuela se presentan en la Tabla I.

TABLA I

CARACTERÍSTICAS DE LA ESCUELA

\begin{tabular}{ll}
\hline \hline Escuela Rural & Santa Bárbara \\
\hline Coordenadas & $32^{\circ} 21^{\prime} 11.81^{\prime \prime S} ; 65^{\circ} 47^{\prime} 25.45^{\prime \prime O}$ \\
Paraje & Puertas del Sol \\
Departamento & San Martín \\
Alumnos promedio & $8-10$ \\
Días de clase & Lunes a Viernes \\
Horarios & 10 a 15 hs. \\
Sup. Cubierta & $64 \mathrm{~m} 2$ \\
Vía & Difícil acceso \\
Docentes & 5 \\
\hline \hline
\end{tabular}

La Fig. 2.a presenta una vista aérea de la ubicación de la escuela Santa Bárbara y la distancia con respecto a las localidades más cercanas.

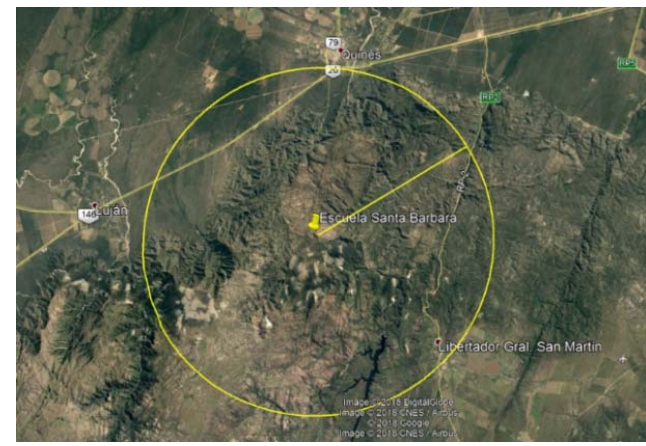

a) Lugar de emplazamiento

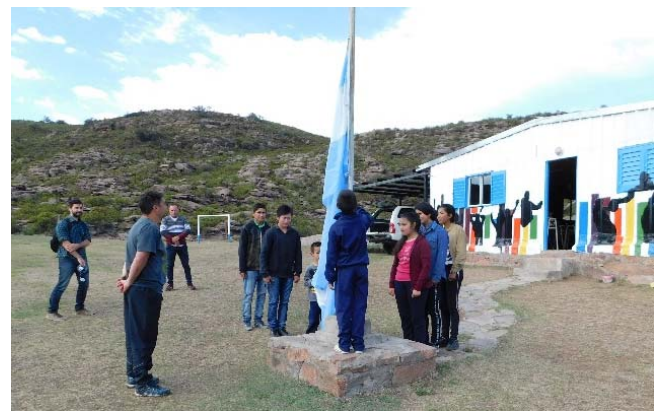

b) Alumnos de la escuela Santa Bárbara

Fig. 2. Imágenes de la Escuela Rural Santa Bárbara.

Como se mencionó en la Sección 2, el primer paso en el diseño de una microrred consiste en la Recopilación de Datos, a continuación se presentan los distintos tipos de fuentes de energía renovables y no renovables disponibles, como también el consumo de potencia eléctrica de la escuela.

\section{A. Recursos Renovables Disponibles}

Para realizar el estudio de recursos renovables (viento e irradiancia) se consultaron diferentes bases de datos. Se tomó como base la provista por el software HOMER Pro que pertenece a la NASA para las coordenadas de la escuela, y mediante bases de datos locales y mediciones realizadas en campo se escalaron en el software. Para la energía eólica se consultó la base de datos provista por el Sistema de
Información Geográfica (SIG) del Ministerio de Energía de la Nación y para la irradiación y temperatura las bases provistas por la Red de Estaciones Meteorológicas (REM) de la Provincia de San Luis. En las Fig. 3 y 4 se presentan los perfiles definitivos cargados en el software.

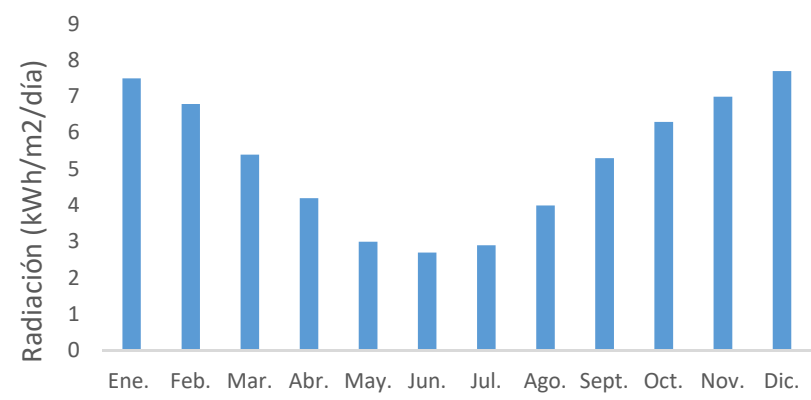

Fig. 3. Perfil anual de irradiancia en $\mathrm{kWh} / \mathrm{m} 2 /$ día.

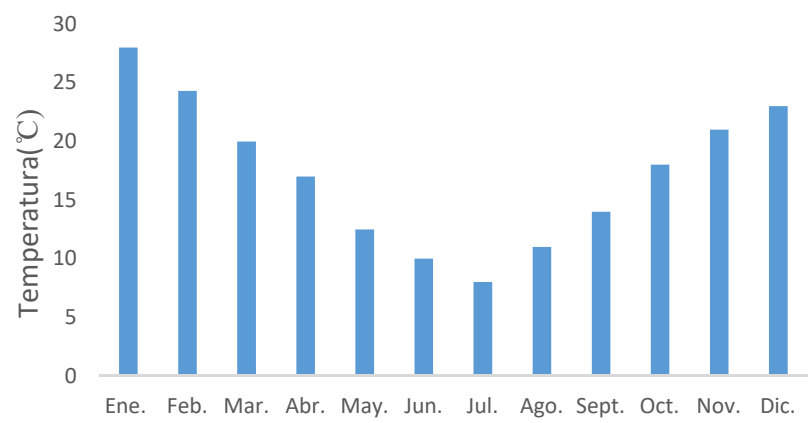

Fig. 4. Perfil anual de temperatura en grados centígrados.

A partir de las Fig. 3 y 4 se configuró un sistema fotovoltaico de $250 \mathrm{~W}$, cuyas especificaciones se detallan en la Tabla IV.

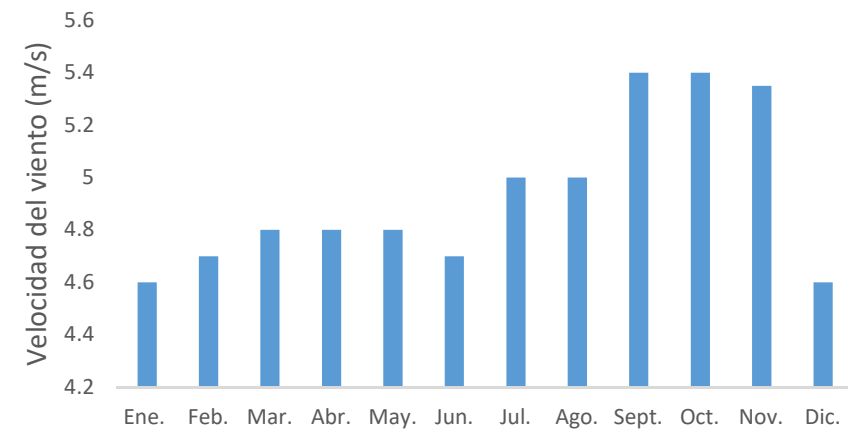

Fig. 5. Perfil anual de velocidad de viento en $\mathrm{m} / \mathrm{s}$

Como se observa en la Fig. 5, la escuela cuenta con buen recurso eólico por lo que se configuró un sistema con un generador de $650 \mathrm{~W}$ genérico. Los detalles de la configuración del sistema eólico se presentan en la Tabla IV.

\section{B. Energías Convencionales}

\section{Grupo Electrógeno}

Se propone un grupo electrógeno como alternativa para los momentos en que tanto las baterías como los recursos renovables no puedan abastecer el consumo de la escuela. Un parámetro crítico de este sistema, es el precio del combustible 
diésel, y el pronóstico de aumento en los próximos años a través del parámetro de inflación que posee el software. Con base en esto se analizó el precio en los últimos 10 años y se cargaron los datos en el software haciendo una estimación de cómo se irá incrementando el valor del combustible.

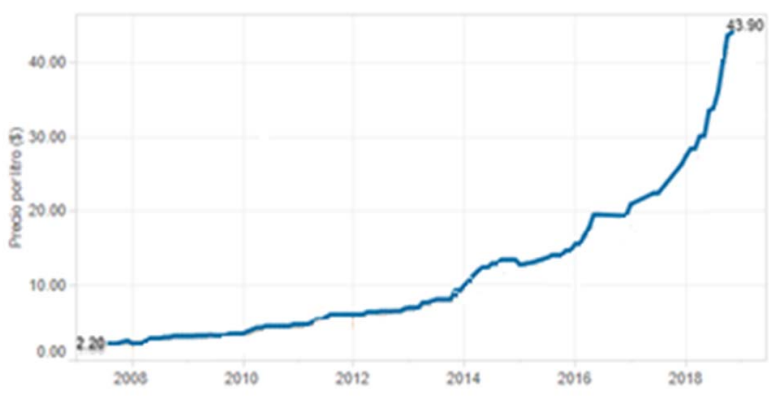

Fig. 6. Precio del combustible diésel en los últimos 10 años en Argentina.

Como se observa el valor de combustible es un parámetro que aumenta año tras año. Además otro factor que complica la utilización del grupo electrógeno es la dificultad de acceso a la escuela rural. Aprovechando que la escuela ya cuenta con uno de estos sistemas, se incorpora un sistema de este tipo al modelo del diseño para su posterior optimización, pensado que su uso solo será el menor tiempo posible.

\section{Interconexión a la Red}

Otra de las opciones analizadas fue la posibilidad de abastecer de potencia eléctrica a la escuela interconectándola con la Red Eléctrica. Para esto se realiza la proyección a una línea disponible en la ruta RP2, que es una línea trifásica coplanar de 13,2 KV. Debido a que es una zona montañosa, hay que emplear explosivos y postes de hormigón encareciendo el costo de la obra.

En la Tabla II se presenta una cotización actual para esta propuesta acordes a lo que maneja actualmente el mercado provista por el Ministerio de Obras Públicas e Infraestructura de la Provincia de San Luis [21].

TABLA II

COTIZACIÓN DEL TENDIDO DE LA RED ELÉCTRICA A LA ESCUELA

\begin{tabular}{llll}
\hline \hline Descripción & Cantidad & C. Unitario & C. Total \\
\hline Permisos (Municipales, Ing.) & 1 & $\$ 161.512$ & $\$ 161.512$ \\
LMT 13,2 kV trif. 35mm2 & $12,8 \mathrm{Km}$ & $\$ 178.372$ & $\$ 2.283 .161$ \\
Estructuras LMT & 40 & $\$ 32.543$ & $\$ 1.301 .720$ \\
Transf. Trif. 16kVA & 1 & $\$ 123.960$ & $\$ 123.960$ \\
LBT 3x25mm2+1x50mm2 & $0.5 \mathrm{Km}$ & $\$ 201.886$ & $\$ 100.943$ \\
Pilar medición & 1 & $\$ 8.541$ & 8.541 \\
Pozeado con explosivos & 150 & $\$ 7.117$ & $\$ 1.067 .550$ \\
\hline Subtotal & & & $\$ 5.047 .387$ \\
Coeficiente impacto & 1,6186 & & \\
Total. & & & $\$ 8.169 .701$ \\
\hline \hline
\end{tabular}

\section{Almacenadores de Energía}

\section{Baterías}

Las fuentes de energía renovables (como la fotovoltaica y eólica), se pueden utilizar en áreas aisladas debido a su característica de autonomía, sin embargo, no pueden asegurar que la potencia entregada a la carga sea ininterrumpida. Es por esto que los sistemas híbridos cuentan con almacenadores de energía para garantizar la continuidad del suministro de energía.

En este estudio, se utiliza el modelo de batería de plomo ácido, que proporciona buenas características combinadas con bajo costo. La vida útil y la efíciencia de la batería se establecen en 5 años y $85 \%$, respectivamente.

\section{Consumo eléctrico}

Las mediciones de campo del consumo de la escuela se realizaron durante varios días y también se utilizó información de entrevistas personales con los docentes de la escuela. Esta información es de vital importancia para el diseño de la microrred para poder realizar una optimización adecuada teniendo en cuenta posibles imprevistos, futuros incrementos de consumo, etc.

A partir de las mediciones de campo realizadas se confeccionó la Tabla III.

TABLA III

CONSUMOS

\begin{tabular}{|c|c|c|c|c|c|c|}
\hline Equipo & No & Watt & $\begin{array}{l}\text { Total } \\
\text { Watt } \\
\end{array}$ & $\begin{array}{l}\text { Horario de } \\
\text { Consumo }\end{array}$ & $\begin{array}{l}\text { Usado } \\
\text { Hr/Día }\end{array}$ & $\begin{array}{l}\text { Watt } \\
\text { Hr./Día }\end{array}$ \\
\hline $\mathrm{PC}$ & 5 & 50 & 250 & 9 a $15 \mathrm{hs}$ & 4 & 1000,0 \\
\hline $\begin{array}{l}\text { Iluminación } \\
\text { Interior }\end{array}$ & 5 & 10 & 50 & 9 a $15 \mathrm{hs}$ & 6 & 300,0 \\
\hline $\begin{array}{l}\text { Iluminación } \\
\text { Exterior }\end{array}$ & 2 & 10 & 20 & 18 a 6 hs & 6 & 120,0 \\
\hline Bomba & 1 & 750 & 750 & 10 a $11 \mathrm{hs}$ & 1 & 750,0 \\
\hline $\begin{array}{l}\text { Antena } \\
\text { WIFI }\end{array}$ & 1 & 55 & 55 & $24 \mathrm{hs}$ & 24 & 1320 \\
\hline Proyector & 1 & 100 & 100 & 9 a 12 hs & 3 & 300,0 \\
\hline Impresora & 1 & 50 & 50 & $10 \mathrm{a} 12 \mathrm{hs}$ & 2 & 100,0 \\
\hline $\begin{array}{l}\text { Equipo de } \\
\text { Música }\end{array}$ & 1 & 50 & 50 & 11 a $15 \mathrm{hs}$ & 4 & 200,0 \\
\hline TV Smart & 1 & 50 & 50 & 11 a $15 \mathrm{hs}$ & 4 & 200,0 \\
\hline Celulares & 4 & 5 & 20 & $\begin{array}{l}9 \text { a } 11 \text { y } 18 \text { a } \\
21 \mathrm{hs}\end{array}$ & 5 & 100,0 \\
\hline Total & & & & & & 4390,0 \\
\hline
\end{tabular}

El perfil de consumo tiene la particularidad que el mayor consumo ocurre durante horarios diurnos que funciona la escuela a diferencia de un consumo característico de una casa convencional, donde el mayor consumo se produce por la noche; esta es una ventaja para maximizar el uso de la energía solar disponible en la zona. También, se tuvo en cuenta los meses que dura el ciclo escolar y los fines de semana donde no hay actividad y el consumo es solamente de la antena satelital WIFI (la cual es utilizada por los pobladores cercanos para comunicarse debido a la falta de señal telefónica). En la Fig. 7 se presenta el perfil de consumo para un día de semana durante el periodo de clases. Se puede observar que el máximo consumo (bomba para carga del tanque de agua) se programa en los horarios donde la irradiancia es máxima.

Las Fig. 8 y 9 presentan el consumo anual de la escuela donde se observa que durante los meses de receso, el consumo es mucho menor y está destinado solo a iluminación exterior por la noche y a la antena satelital WIFI. Además, se observa 
que el pico de consumo es cuando la bomba de agua es activada para cargar el tanque de la escuela.

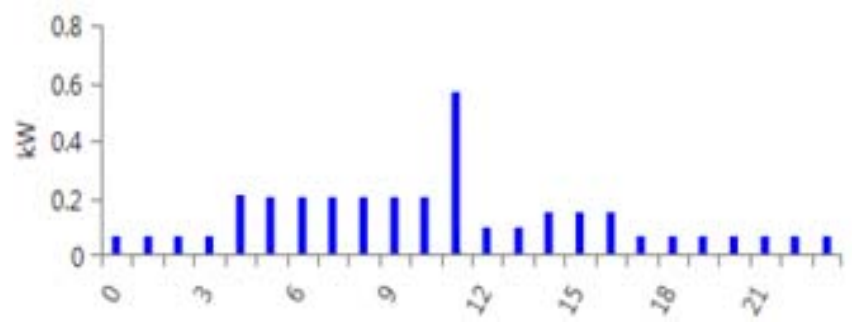

Fig. 7. Perfil de consumo diario.

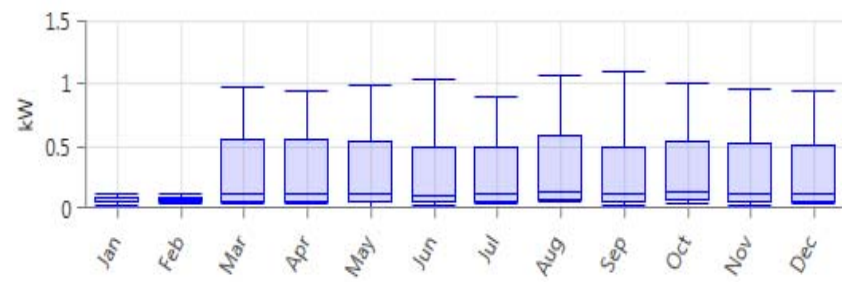

Fig. 8. Perfil de consumo anual.

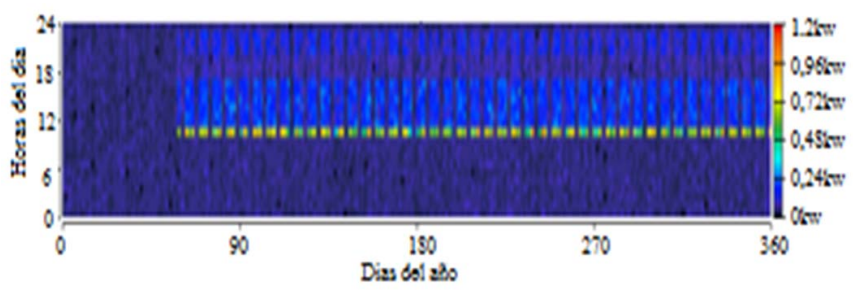

Fig. 9. Perfil de consumo diario y anual.

Una vez concluida la Recopilación de datos, el siguiente paso consiste en la definición de la Topología y Componentes, que será presentado en la siguiente subsección.

\section{E. Configuración de HOMER Pro}

El procedimiento para realizar un proyecto en HOMER Pro consiste en localizar las coordenadas del punto de emplazamiento donde se ubicará el sistema. Mediante la localización es posible actualizar las bases de los recursos disponibles como viento, temperatura e irradiación, entre otros. Luego se procede a configurar cada uno de los sistemas que se quieren evaluar, para nuestro caso de estudio. La Fig. 10 presenta un diagrama de la microrred que será evaluada en este trabajo.

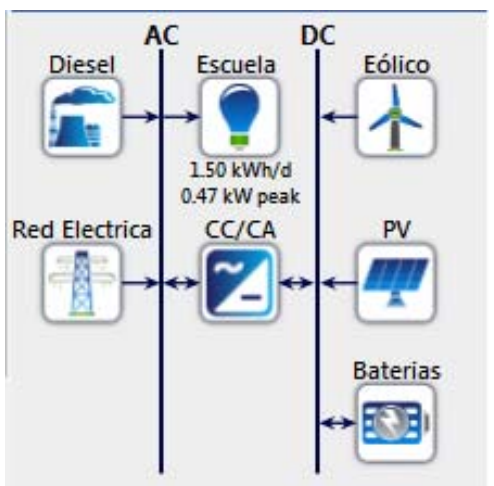

Fig. 10. Diagrama del sistema simulado.
En la Tabla IV se detallan todos los componentes seleccionados con las características y costos que poseen (https://enertik.com.ar/). También, se define el espacio de búsqueda que permite acotar las opciones para que se realice la optimización. Estos espacios de búsqueda son definidos por el software de diferente manera para cada componente y pueden ser en potencia (kW) o unidades (un). La correcta selección de estos espacios impactara directamente en los tiempos de simulación, reduciendo o incrementando el número de iteraciones en la optimización ya que el software considera todas las combinaciones posibles.

Con esta información, el software es capaz de realizar la simulación y encontrar la mejor opción basándose en una optimización económica.

TABLA IV

ESPECIFICACIONES DE LA MICRORRED

\begin{tabular}{|c|c|c|}
\hline Sistema & Especificación & Descripción \\
\hline $\begin{array}{l}\text { Configuración } \\
\text { General }\end{array}$ & $\begin{array}{l}\text { Tasa de Descuento } \\
\text { Tasa Anual de Escasez } \\
\text { Tasa de Inflación } \\
\text { Tiempo de Vida del Proyecto } \\
\text { \% Mínimo de Renovable }\end{array}$ & $\begin{array}{l}8 \% \\
0 \\
10 \% \\
25 \text { años } \\
50 \%\end{array}$ \\
\hline $\begin{array}{l}\text { Sistema } \\
\text { Fotovoltaico }\end{array}$ & $\begin{array}{l}\text { Tamaño (espacio de } \\
\text { búsqueda) } \\
\text { Costo de capital }(1 \times 250 \mathrm{~W}) \\
\text { Costo de reemplazo } \\
\text { Costo de O\&M } \\
\text { Tiempo de vida de } \\
\% \text { de degradación }\end{array}$ & $\begin{array}{l}0-0,25-0,50- \\
0,75-1 \mathrm{~kW} \\
\$ 10000 \\
\$ 10000 \\
\$ 100 \\
25 \text { años } \\
80 \%\end{array}$ \\
\hline Sistema eólico & $\begin{array}{l}\text { Tamaño (espacio de } \\
\text { búsqueda) } \\
\text { Costo de Capital ( } 650 \mathrm{~W}) \\
\text { Costo de Reemplazo } \\
\text { Costo de O\&M } \\
\text { Tiempo de Vida }\end{array}$ & $\begin{array}{l}0-1-2 \text { unidades } \\
\$ 30.000 \\
\$ 30.000 \\
\$ 20 \\
20 \text { años }\end{array}$ \\
\hline Sistema Diésel & $\begin{array}{l}\text { Tamaño (espacio de } \\
\text { búsqueda) } \\
\text { Costo de Capital }(1 \mathrm{~kW}) \\
\text { Costo de Reemplazo } \\
\text { Costo de O\&M } \\
\text { Tiempo de vida }\end{array}$ & $\begin{array}{l}1-2-3-4-5 \mathrm{~kW} \\
10000 \\
10000 \\
\$ 100 \\
15000 \text { horas }\end{array}$ \\
\hline Batería & $\begin{array}{l}\text { Tamaño (espacio de } \\
\text { búsqueda) } \\
\text { Voltaje de batería } \\
\text { Tamaño del string } \\
\text { Capacidad Nominal } \\
\text { Capacidad Máxima } \\
\text { Costo de Capital } \\
\text { Costo de Reemplazo } \\
\text { Costo de O\&M } \\
\text { Tiempo de Vida }\end{array}$ & $\begin{array}{l}0-2-4-8-16 \\
\text { unidades } \\
12 \mathrm{~V} \\
2(24 \mathrm{~V}) \\
1 \mathrm{kWh} \\
83,4 \mathrm{Ah} \\
\$ 7000 \\
\$ 7000 \\
\$ 10 \\
5 \text { años }\end{array}$ \\
\hline $\begin{array}{l}\text { Conexión a la } \\
\text { Red }\end{array}$ & $\begin{array}{l}\text { Costo del capital } \\
\text { Distancia hasta la red } \\
\text { O\&M } \\
\text { Precio de la energía }\end{array}$ & $\begin{array}{l}500.000 \$ / \mathrm{km} \\
15 \mathrm{~km} \\
\$ 160 \\
0,1 \$ / \mathrm{kWh}\end{array}$ \\
\hline
\end{tabular}

Donde puede observarse de la Tabla V que en el software solo se incluye "\% de degradación" para los paneles fotovoltaicos. 


\section{RESUltados OBtenidos}

Una vez realizada la simulación y la optimización, los resultados entregan todas las configuraciones posibles con diferentes valores de potencia y pueden ser ordenados en función de diferentes parámetros, generalmente y como se mencionó en la Sección 2, la configuración con menor costo neto total $\left(\mathrm{C}_{\mathrm{NPC}}\right)$ es la óptima. También se puede observar que el valor del COE es el mínimo para la configuración seleccionada.

Para nuestro caso de estudio, es posible observar en la Fig. 11.a que la configuración con menor $\mathrm{C}_{\mathrm{NPC}}$ está conformada por un sistema fotovoltaico de $0,5 \mathrm{~kW}$ ( 2 paneles), un sistema eólico de $650 \mathrm{~W}$, un grupo electrógeno de $1.3 \mathrm{~kW}, 2$ baterías en paralelo $(24 \mathrm{~V})$ y un convertidor $\mathrm{CC} / \mathrm{CA}$ de $650 \mathrm{~W}$. La misma esta seleccionada (recuadro verde) en la Fig. 11.a.

Una aclaración importante es que si bien el convertidor y los sistemas eólicos y fotovoltaicos están sobredimensionados, estos son las potencias mínimas disponibles en la base de datos del software.

\section{A. Análisis Económico}

En la Fig. 11.b se presentan los costos totales del proyecto considerando el capital inicial, los reemplazos, el mantenimiento, el valor de desecho y el combustible utilizado.

\begin{tabular}{|c|c|c|c|c|c|c|c|c|c|c|c|c|c|c|c|c|}
\hline \multicolumn{11}{|c|}{ Avchitecture } & \multicolumn{4}{|c|}{ Cost } & \multicolumn{2}{|c|}{ System } \\
\hline$\Delta$ & $7+$ & $\overline{1}$ & c3 & $1 \mathrm{Z}$ & ${ }_{\mathrm{WW}}^{\mathrm{PV}} \mathrm{P}$ & Ene0.5 Y & $\begin{array}{l}\text { Gen } ? \\
\operatorname{cic} ?\end{array}$ & IKWMLA 8 & $\begin{array}{c}\text { Conent } 5 W 2524 \\
\mathrm{nW}\end{array}$ & Dapatch & ${ }_{\text {in }}^{C O E} \mathrm{P}$ & ${ }_{(5)}^{N P C}$ \& 8 & $\begin{array}{c}\text { Operating cost } \\
\text { (Shat }\end{array}$ & $\begin{array}{c}\text { Intial capital } \\
\text { (f) }\end{array}$ & $\begin{array}{c}\text { Ren frac } 08 \\
\mathrm{Bg}\end{array}$ & $\begin{array}{c}\text { Total Fuel } \\
\text { Month }\end{array}$ \\
\hline & $7+$ & 5 & $\mathbf{0}$ & $F$ & 0.500 & 1 & 130 & 2 & 0.625 & LF & 5465 & 5597,256 & 53,881 & 595.500 & 91.5 & 402 \\
\hline & $t$ & i & $\mathbf{0}$ & $z$ & & 1 & 130 & 2 & 0359 & LF & 58.59 & 51.10M & 57,964 & 570.188 & 724 & 130 \\
\hline & 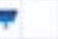 & $\mathbf{E}$ & 0 & Z & 0.500 & & 130 & 4 & 0.313 & LF & $\$ 1102$ & S1.A1M & $\$ 10,326$ & 579.250 & 75.3 & 219 \\
\hline & & $\bar{z}$ & 6 & Z & & & 130 & 4 & 0.281 & if & 533.21 & $\$ 4.25 \mathrm{M}$ & 532504 & 558,625 & 0 & 613 \\
\hline & $\Rightarrow+$ & -5 & & $\boldsymbol{F}$ & 0.500 & 1 & 1.30 & & 0.354 & $\mathrm{cc}$ & $\$ 44.43$ & $\$ 5.70 \mathrm{M}$ & $\$ 43,560$ & 570,083 & 0 & 978 \\
\hline & t & -5 & & 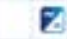 & & 1 & 130 & & 0.250 & $\mathrm{cc}$ & $\$ 49.99$ & $\$ 6.42 \mathrm{M}$ & $\$ 49.249$ & $\$ 48000$ & 0 & 1,106 \\
\hline
\end{tabular}

a). Lista de configuraciones optimas luego de la simulación

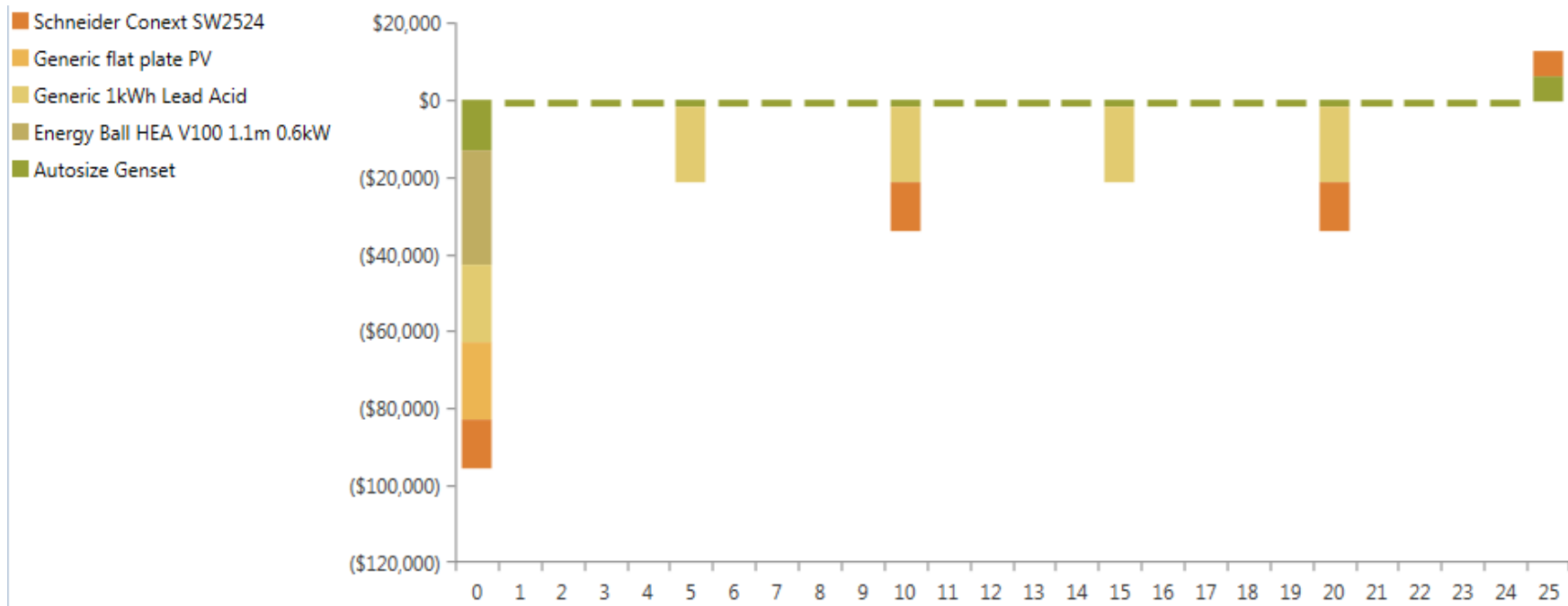

b). Flujo de dinero en los 25 años de duración del proyecto

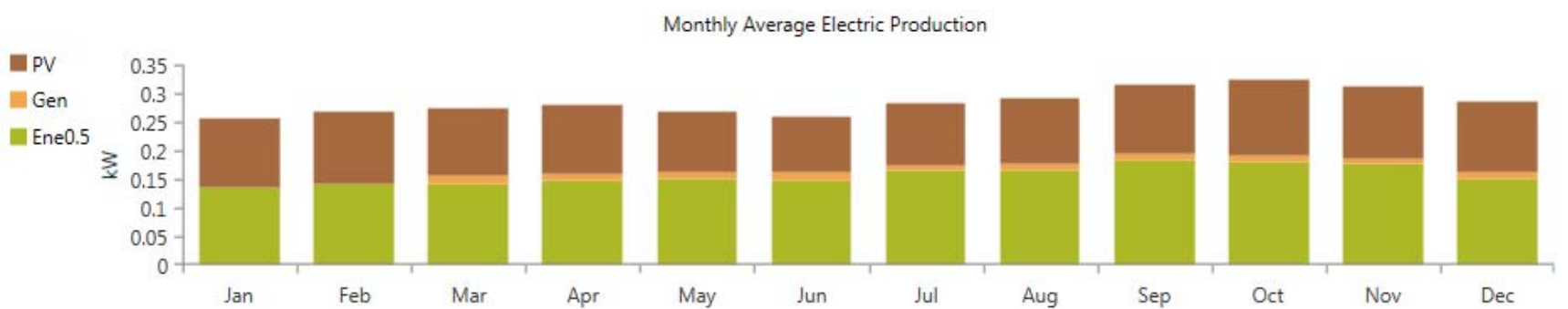

c). Distribución de la producción de energía de la microrred.

Fig. 11 Resultados de Simulación.

La Fig. 11.b muestra el flujo de dinero a lo largo de 25 años de duración del proyecto, se debe destacar que el costo más importante es el costo de capital inicial (característico de los sistemas basados en energías renovables), y que luego tiene un costo considerablemente menores en periodos de 5 años y 10 años, debido al recambio de baterías y del convertidor respectivamente, más un costo fijo menor del grupo electrógeno. Una vez finalizado el tiempo de vida del 
proyecto, el resultado es un costo de recuperación, que básicamente es el tiempo que podría seguir funcionando, en este caso, el inversor y el grupo electrógeno.

En la Fig. 11.c se presenta el análisis de la producción de cada uno de los sistemas de generación. Se observa que el generador eólico proporciona el $55,1 \%$ de la potencia mientras que el sistema fotovoltaico el $41,6 \%$, la potencia restante es provista por el grupo electrógeno con un $3.3 \%$.

En la Fig. 12 se presenta una comparación entre el sistema aislado y conectado a la red. Se observa el costo en función de la distancia de la línea de transmisión hasta la escuela. Se puede observar que para los valores presupuestados, a los 1,12 $\mathrm{km}$ ya se igualan los costos de ambos sistemas, concluyendo que el conectar la escuela a la red eléctrica sería muy costoso en comparación con el sistema aislado.

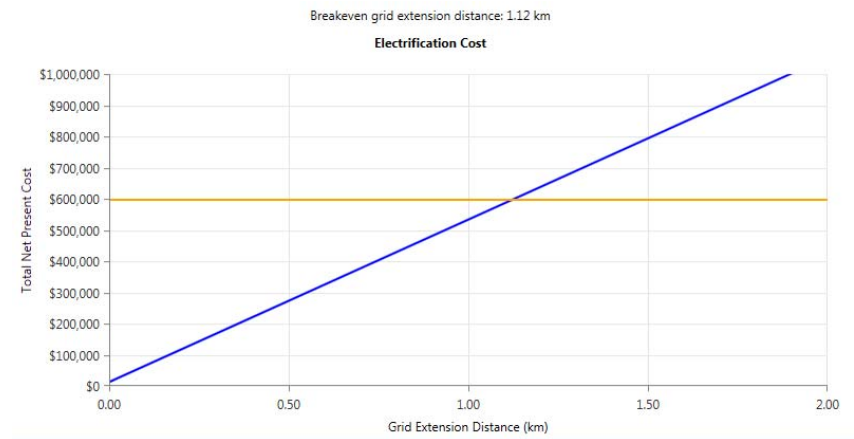

Fig. 12. Comparación del costo entre el sistema aislado y conectado a la red.

\section{B. Análisis Energético}

En las Fig. 13-16 se presentan cada sistema de generación por separado.

El sistema fotovoltaico, conformado por dos paneles fotovoltaicos, en condiciones óptimas, entrega $1035 \mathrm{kWh} /$ año. Otra ventaja del sistema fotovoltaico que presenta la Fig. 13, es que la máxima generación se produce entre las 10:00 y 16:00 hs como es de esperarse, y coincide con los periodos más altos de consumo de la escuela.

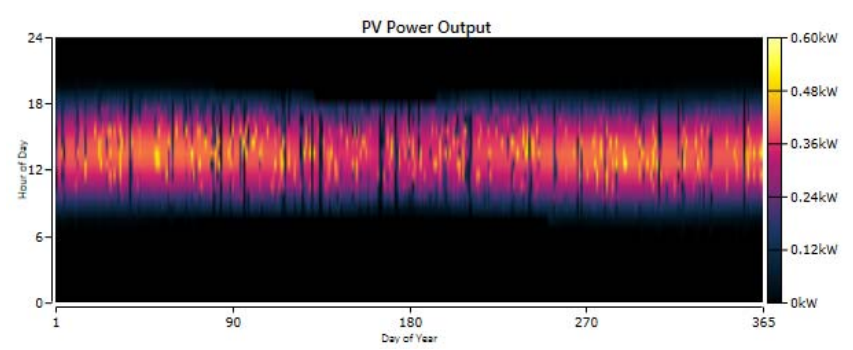

Fig. 13. Producción de energía anual del sistema fotovoltaico.

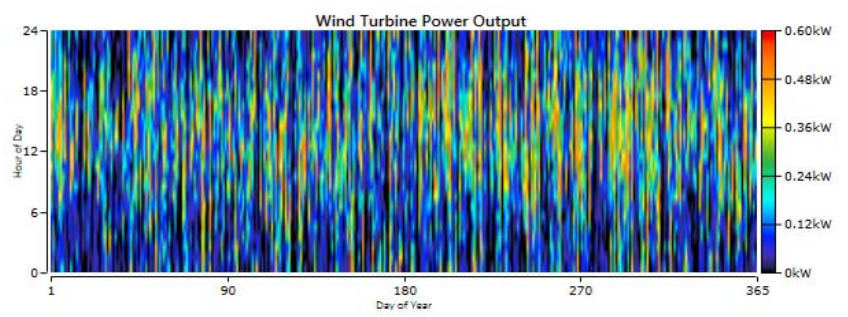

Fig. 14. Producción de energía anual del sistema eólico.
El sistema eólico es la fuente renovable que mayor energía entrega a la microrred con $1371 \mathrm{kWh} / \mathrm{año}$. Su comportamiento es estocástico aunque las mayores épocas de viento se dan entre julio y noviembre.

Del sistema propuesto se puede observar en la Fig. 15 que el estado de carga de las baterías oscila entre los rangos de carga configurados, nunca descendiendo del $40 \%$ de estado de carga preestablecido.

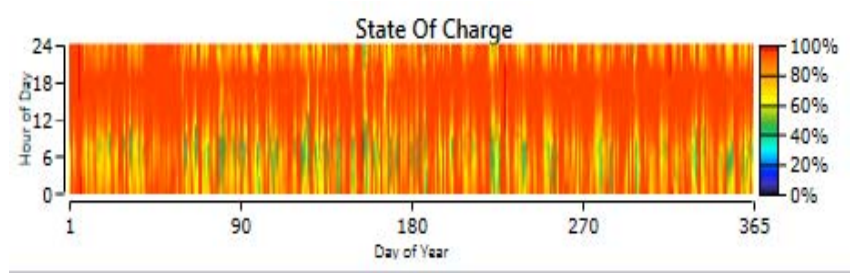

Fig. 15. Estado de carga del almacenamiento de energía.

Como se mencionó anteriormente, el sistema diésel se utilizará un porcentaje reducido durante el año. En la Fig. 16 se observa que principalmente los momentos donde funciona el grupo electrógeno son los instantes donde la bomba de agua está trabajando y realizando la carga del tanque de agua.

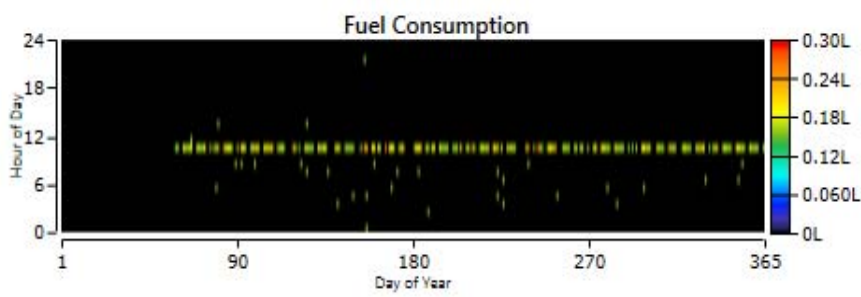

Fig. 16. Consumo de combustible del grupo electrógeno.

\section{Comparación con un Sistema Tradicional}

Un análisis muy importante es la comparación del sistema elegido con un sistema tradicional basado en solamente contar con un grupo electrógeno. En la Tabla $\mathrm{V}$ se observa que si bien el costo del capital inicial es mucho menor que el sistema propuesto (86,3\% menos), el costo total del sistema a lo largo de los 25 años es un 93\% mayor, básicamente debido a los gastos fijos de combustible.

TABLA V

COMPARACIÓN DE COSTOS DE SISTEMAS AISLADOS

\begin{tabular}{lll}
\hline \hline Sistema & Costo inicial & Costo total \\
\hline Híbrido & $\$ 95.500$ & $\$ 597.256$ \\
G. Electrógeno & $\$ 13.000$ & $\$ 8.640 .000$ \\
\hline \hline
\end{tabular}

En la Fig. 17 se presenta el flujo de fondos de ambos sistemas, se observa que el sistema elegido posee un gran costo inicial pero el grupo electrógeno posee un costo anual de $\$ 70.000$ pesos en combustible.

\section{CONCLUSIONES}

En este trabajo se propuso una metodología compuesta por cuatro etapas simples, que permiten el dimensionamiento y selección de componentes óptimos para microrredes basadas en energías renovables en zonas remotas o aisladas de la red. 

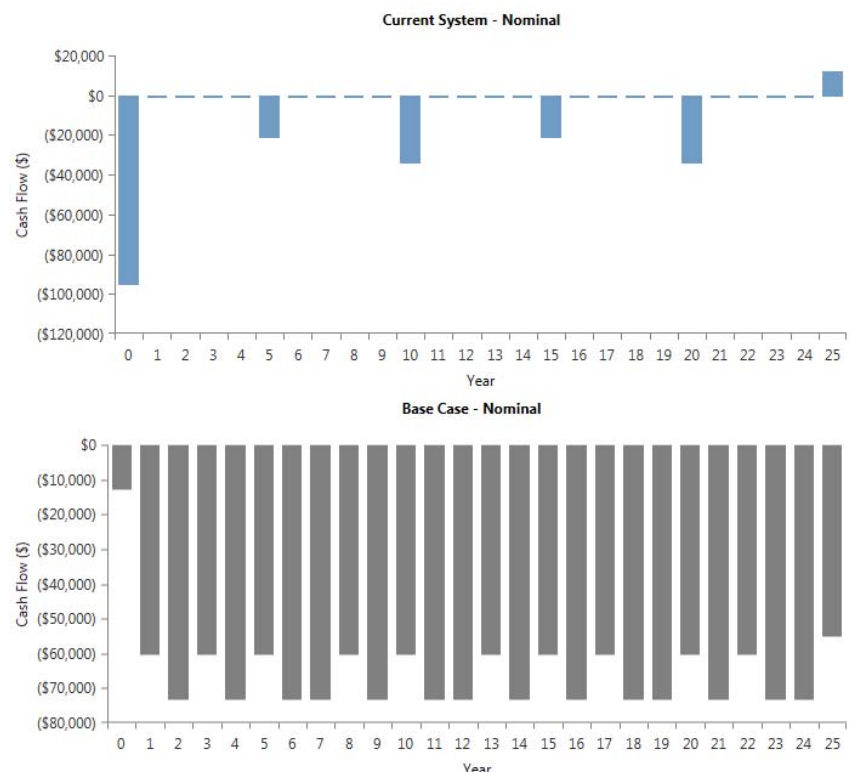

Fig. 17. Comparación del flujo de fondos del sistema híbrido y grupo electrógeno.

Para el caso de estudio analizado, la metodología basada en criterios económicos concluyó que la mejor opción es un sistema híbrido conformado por un sistema eólico, fotovoltaico y baterías para el almacenamiento. Además, este sistema considera un grupo electrógeno como reserva en casos donde la microrred no pueda abastecer el consumo total.

La principal ventaja de la microred es que proveerá del servicio de internet las 24 hs, lo que se traduce no sólo como una vía más efectiva de comunicación (fundamental para el pedido de asistencia frente a posible riesgo de la población), sino que también posibilita a una comercialización más óptima de los productos (la mayoría libre de conservantes) realizados en el lugar.

También la incorporación de energías renovables disminuirá los costos actuales de combustible y reducirá los porcentajes de contaminación.

Con la base de este trabajo, se espera continuar trabajando en metodologías de dimensionamiento de microrredes aisladas incorporando nuevos criterios como ambientales y sociales para evaluarlos con técnicas multicriterio.

\section{AgRadecimientos}

Este trabajo ha sido realizado parcialmente gracias al apoyo de la Secretaría de Políticas Universitarias (SPU) con el proyecto "Microrred para una escuela rural" de la convocatoria Agregando Valor y a la red CYTEDMEIHAPER.

\section{REFERENCIAS}

[1] Goel, S., Sharma, R., 2017. Performance evaluation of stand alone, grid connected and hybrid renewable energy systems for rural application: a comparative review. Renew. Sustain. Energy Rev. 78(October), $1378-$ 1389.

[2] Khodayar, M.E. Rural electrification and expansion planning of off-grid microgrids. Electr. J. 2017, 30, 68-74.

[3] A. Chauhan and R: Saini, "A review on Integrated Renewable Energy System based power generation for standalone applications: Configurations, storage options, sizing methodologies and control", Renewable and Sustainable Energy Reviews, 2014, vol. 38, pp. 99-129.
[4] O. Erdinc and M. Uzunoglu, "Optimum design of hybrid renewable energy systems: Overview of different approaches", Renewable and Sustainable Energy Reviews, 2012, vol. 16, pp. 1412-1425.

[5] D. Feroldi and D. Zumoffen, "Sizing methodology for hybrid systems based on multiple renewable power sources integrated to the energy management strategy", International Journal of Hydrogen Energy, 2014, vol. 39, pp. 8609-8620.

[6] J. Goncalves, J. Mendes, M. Resende, "A genetic algorithm for the resource constrained multi-project scheduling problem", European Journal of Operational Research, 2008, 189(3):1171-90.

[7] C. Bordons, F. García-Torres, L. Valverde, "Gestión Óptima de la Energía en Microrredes con Generación Renovable" Revista Iberoamericana de Automática e Informática industrial, 2015, vol. 12, 117-132.

[8] A. Lorestani, G.B. Gharehpetian, M. H. Nazari, "Optimal sizing and techno-economic analysis of energy- and costefficient standalone multicarrier microgrid" Energy, 2019, vol. 178, pp: 751-764.

[9] C. Gamarra, J. M. Guerrero "Computational optimization techniques applied to microgrids planning: a review", Renew Sustain.Energy Rev, 48 (2015), pp.413-424.

[10] D. Connolly, H. Lund, B. Mathiesen and M. Leahy, "A review of computer tools for analysing the integration of renewable energy into various energy systems" Applied Energy, 2010, vol. 87 (4):1059-82.

[11] T. Lambert, P. Gilman and P. Lilienthal, "Micropower system modeling with HOMER" In: Farret, F. and Simoes M., Integration of Alternative Sources of Energy, 2006, John Wiley \& Sons, Inc., USA, Ch. 15, pp 379418.

[12] S. Silva, M. Severino and M. De Oliveira "Sizing and Optimization of Hybrid Photovoltaic, Fuel Cell and Battery System" IEEE Latin America Transactions, 2011, vol. 9, Issue: 1, pp $817-822$.

[13] D. W. Gao, Energy Storage For Sustainable Microgrid, London, U.K.:Elsevier, 2015.

[14] F. Ahmad, M. S. Alam, "Economic and ecological aspects for microgrids deployment in India" Sustain. Cities Soc., vol. 37, pp. 407-419, Feb. 2018.

[15] A Nespoli, E Ogliari, S Leva, A Massi Pavan, A Mellit "Dayahead photovoltaic forecasting: A comparison of the most effective techniques" Energies, 2019, vol. 12, https://doi.org/10.3390/en12091621.

[16] H. Malik, V. Padmanabhan, R. Sharma "PSO-NN-Based Hybrid Model for Long-Term Wind Speed Prediction: A Study on 67 Cities of India" in Applications of Artificial Intelligence Techniques in Engineering, Volume 2, Springer, 2018.

[17] M. K. Deshmukh, A. B. Singh, "Modeling of Energy Performance of Stand-Alone SPV System Using HOMER Pro", Energy Procedia, 2018 5th International Conference on Power and Energy Systems Engineering, CPESE 2018, vol. 156, pp. 90-94, Nagoya, Japan.

[18] M. Ghiasi,"Detailed study, multi-objective optimization, and design of an AC-DC smart microgrid with hybrid renewable energy resources", Energy, 2019, Volume 169, Pages 496-507.

[19] Barro, R.J., 1997, Macroeconomics (5th edition) Cambridge. The MIT Press. ISBN 0262024365.

[20] HOMER Pro Microgrid Analysis Tool (version 3.7.6) Energy. Available at http://www.homerenergy.com/, accessed 22 October 2017

[21] Ministerio de Obras Públicas e Infraestructura de la provincia de San Luis http://www.obraspublicas.sanluis.gov.ar/

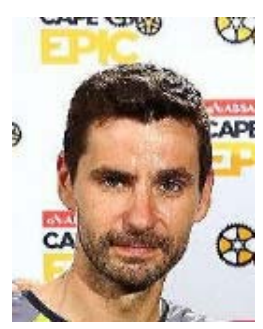

Guillermo R. Catuogno was born in Villa Mercedes, Argentina, in 1981. Received the Electrical and Electronics Engineering degree from Universidad Nacional de San Luis in 2007 and his doctorate of engineering degree from the Universidad Nacional de Río Cuarto in 2013.

Since 2013, he has been with the Universidad Nacional de San Luis, Argentina and CONICET, where he is currently the CoDirector of the Laboratorio de Control Automático. 


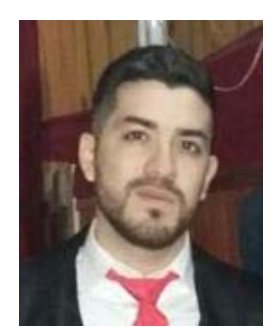

Luis R. Torres was born in Villa Mercedes, San Luis, Argentina, in 1984. He received the title of electronic engineer in 2016 at the Universidad Nacional de San Luis, Argentina. From 2016 to the present, he is doing her doctorate at the Universidad Nacional de San Luis, Argentina, where she is currently an exclusive teacher and member of the Laboratorio de Control Automático.

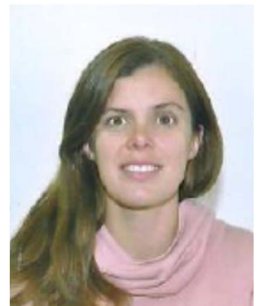

Luciana Proietti was born in Bell Ville, Argentina, in 1981. He graduated in Psychology at the Universidad Siglo 21, Córdoba, Argentina.

Since 2010, she is the co-founder of the NGO 500RPM dedicated to the installation of low power wind turbines in rural areas and since 2016. She is the fundraiser of the Wind Empowerment organization.

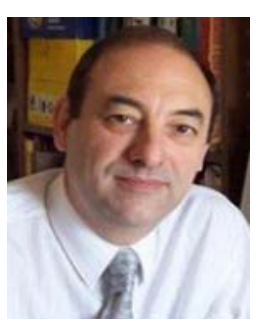

Guillermo O. Garcia was born in Río Cuarto, Argentina, in 1954. Received the Electrical and Electronics Engineering degree from the Universidad Nacional de Córdoba, Córdoba, Argentina, in 1981, and the M.Sc. and Dr. degrees in electrical engineering from COPPE, Universidade Federal do Rio de Janeiro, Rio de Janeiro, Brazil, in 1990 and 1994, respectively. Since 1994, he has been with the Universidad Nacional de Río Cuarto, Río Cuarto, Argentina, where he is currently the Director of the Grupo de Electrónica Aplicada. He is also with the Consejo Nacional de Investigaciones Científicas y Técnicas, Argentina. 